\title{
Case series on remembering pachydermoperiostosis
}

\begin{abstract}
Pachydermoperiostosis is a rare, autosomal dominant clinical condition characterized by progressive enlargement of hands and feet, which can be surmised as acromegaly. We described pachydermoperiostosis in two families and also we shared the long-term follow up results of patients treated with colchicine, acemetacin, and bisphosphonate.
\end{abstract}

Keywords: pachydermoperiostosis, acromegaly, hypertrophic osteoarthropathies

\author{
Volume 10 Issue 2 - 2018
}

\author{
Ilker Ilhanli,' Fazil Kulakli,' Sertac Ketenci, ${ }^{2}$ \\ Elif Uysal,' Adem Turkoz,' Samet Tatli,' Canan \\ Celik \\ 'Department of Physical Medicine and Rehabilitation, School of \\ Medicine, Giresun University, Turkey \\ ${ }^{2}$ Department of Rheumatology, Giresun University, M. Ilhan \\ OZDEMIR Training and Research Hospital,Turkey
}

Correspondence: Ilker ILHANLI, Department of Physical Medicine and Rehabilitation, School of Medicine, Giresun University, 28200, Giresun, Turkey, Tel +90 45431016 90, Fax +904543101699, Email ilkerilhanli@hotmail.com

Received: January 23, 2018 | Published: March 20, 2018

\section{Introduction}

A rare clinical entity pachydermoperiostosis is an autosomal dominant clinical disorder. It involves the distal extremities. The periosteal new bone formation is the characteristic of pachydermoperiostosis. Generally, patients present with clubbing of fingers, progressive enlargement of distal extremities, thickening of the skin and hyperhidrosis. Symptoms usually occur during adolescence. Often patients' symptoms get worse over the next decade and then calm down. Accidentally, the progressive expansion of the hands and feet can be considered acromegaly.

\section{Case report}

\section{Case I}

A 26-year-old male presented to the outpatient clinic because of expansion of his hands and feet since the age of 12 (Figure 1). With widespread pain and swelling in both ankles and knees. Expansion of his hands was insidious in onset and associated with excessive sweating. Positive family history of same complaint in milder degree in his father and elder sister. He had another healthy sister and brother. There was a kinship between the patient's parents. In physical examination, there was severe clubbing of all fingers and toes with enlarged distal extremities. The skin of both hands and feet was thickened. He had arthritis in both knees and ankles. X-ray images showed hypertrophic osteoarthropathy and acroosteolysis (Figure 2). We confirmed the synovitis by magnetic resonance imaging of the knee. Complete blood count, serum glucose, alkaline phosphatase, calcium and phosphorous were normal. Serum growth hormone (GH), insulin-like growth factor 1 (IGF1), thyroid stimulating hormone, free thyroxine 4, prolactin, testosterone, adrenocorticotrophic hormone, and cortisol were normal. Sedimentation rate (ESR) and C - reactive protein were found to be high $(104 \mathrm{~mm} / \mathrm{h}, 21 \mathrm{mg} / \mathrm{L}$, respectively). The patient was diagnosed as pachydermoperiostosis after exclusion of excessive GH production. Treatment including colchicine, acemetacin, and biphosphonate was initiated. Arthritis was improved dramatically, and remarkably patient's symptoms were calm down in a short period.

\section{Case 2}

Elder sister of case 1, a 32-year-old patient who had milder clubbing of all fingers with enlarged distal extremities referred to our clinic. She had no arthritis. All laboratory tests were normal. Radiological examinations showed only hypertrophic osteoarthropathy.

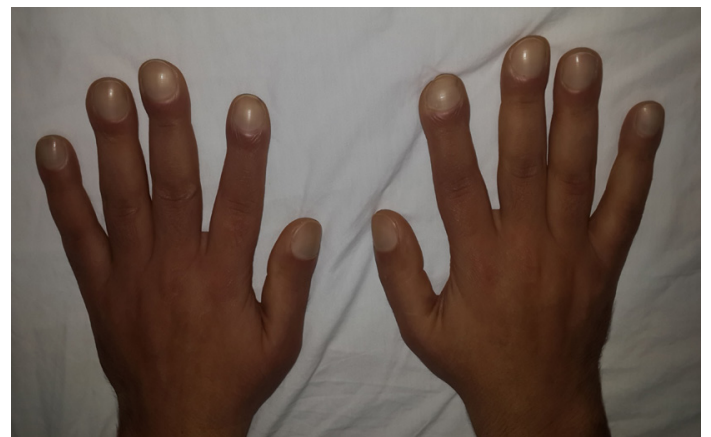

Figure I 26 years old patient expansion of hands and feet since the age of 12 .

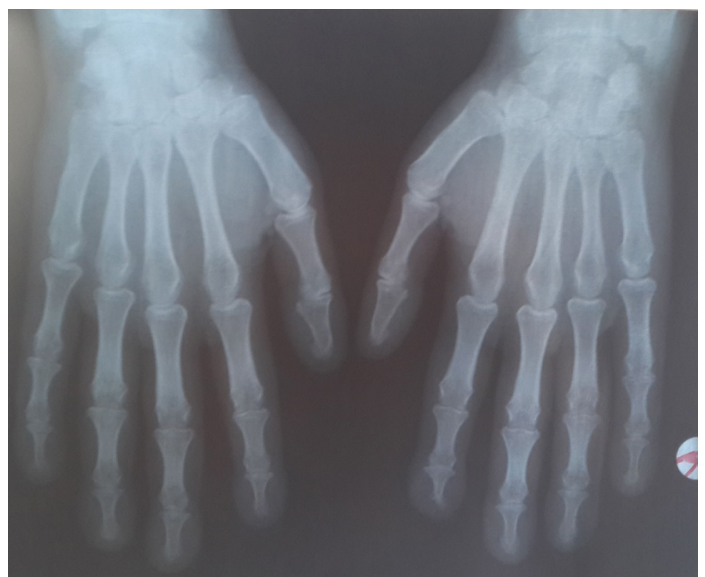

Figure 2 X-ray images showed hypertrophic osteoarthropathy and acroosteolysis.

\section{Case 3}

Father of case 1 and 2, a 56-year-old patient had only milder clubbing of all fingers with enlarged distal extremities and without arthritis. Similar to case 2 his laboratory tests were normal and had only hypertrophic osteoarthropathy in x-ray images. 
Both case 2 and 3 noticed that enlargement was insidious in onset, and got worse over the years but calmed down. Treatment was not started to these patients after discussing the situation with them.

\section{Case 4}

55-year-old female referred to our outpatient clinic with left drop foot after a lumbar surgical intervention. She had arthritis of the left ankle. Also, she was suffering from the expansion of her hands (Figure 3). She had total knee replacement of both knees 11 years ago. She emphasized that enlargement was insidious in onset. She had thickened skin with excessive sweating in both hands and severe clubbing of all fingers. Her son had had similar complaints. X-ray showed hypertrophic osteoarthropathy. All laboratory tests were normal except the increased sedimentation rate (ESR) and C - reactive protein levels $(60 \mathrm{~mm} / \mathrm{h}, 2.71 \mathrm{mg} / \mathrm{L}$, respectively). After exclusion of GH overproduction, the patient was diagnosed as pachydermoperiostosis. Combination therapy of colchicine, acemetacin, and bisphosphonate was started. Arthritis and acute phase reactants reduced dramatically, and her symptoms improved remarkably in a short period. Muscle strength of left leg improved after rehabilitation program and dorsiflexion of left ankle increased to $3 / 5$ (according to Medical Research Council).

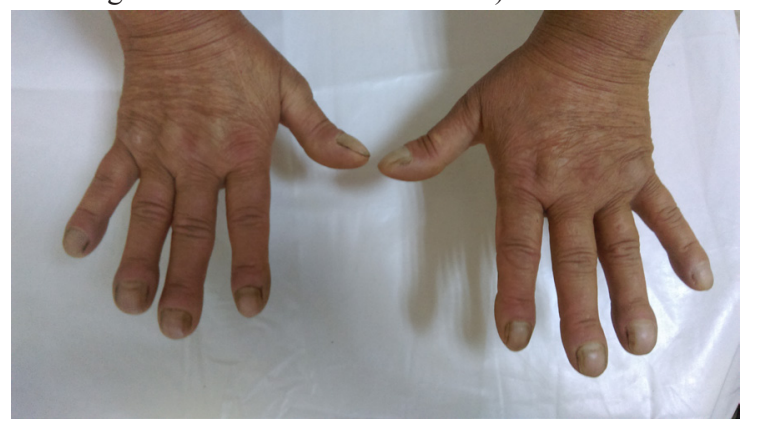

Figure 3 55-year-old female patient suffering from the expansion of her hands.

\section{Case 5}

Son of case 4, 36-year-old patient referred to our outpatient clinic with the complaint of expansion of hands (Figure 4). He had severe widespread joint pain. Visual analog scale (VAS) for pain was 8 points. There was no other evidence of arthritis. Similar to other patients, he mentioned that enlargement was insidious in onset and got worse over the years, until now. All laboratory tests including acute phase reactants were normal. X-ray showed hypertrophic osteoarthropathy and acro-osteolysis (Figure 5). Combination of colchicine, acemetacin, and bisphosphonate was started. His symptoms reduced remarkably in a few weeks. Control VAS was 1 point.

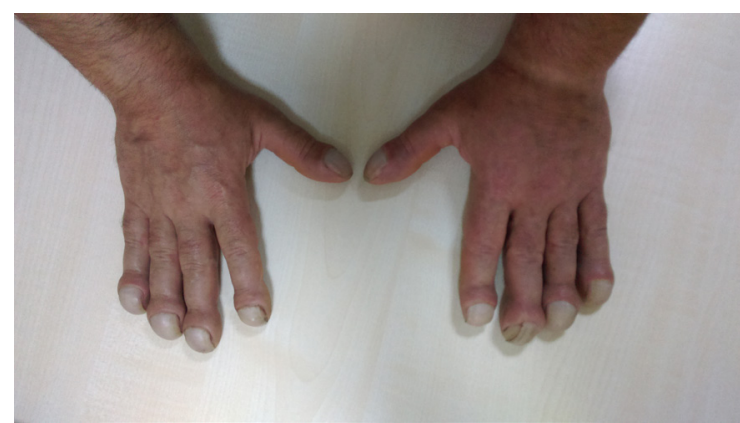

Figure 4 36-year-old patient expansion of hands.

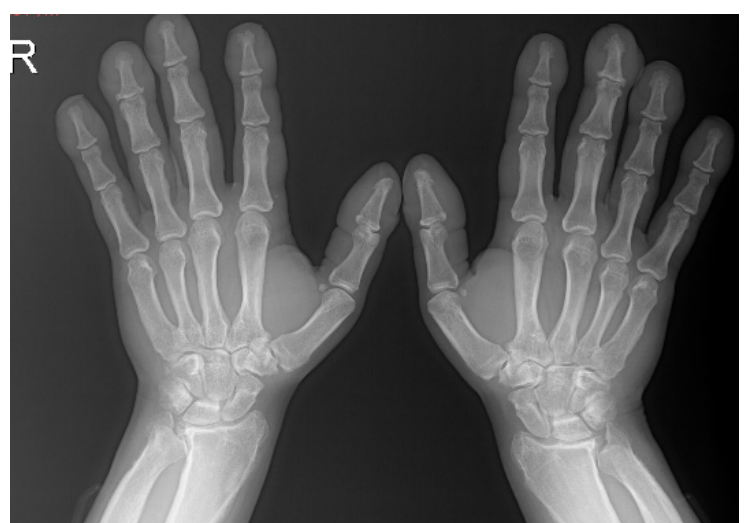

Figure 5 X-ray showed hypertrophic osteoarthropathy and acro-osteolysis

\section{Discussion}

Pachydermoperiostosis was first described in 1868 by Friedrich in two young brothers. Inheritance of this disease is an autosomal dominant trait with variable expression. Positive family history is available in one-third of this patients. ${ }^{2}$ Pachydermoperiostosis differs from the most of well-known secondary hypertrophic osteoarthropathies that are always associated with heart and lung diseases. Onset usually in adolescence and symptoms frequently get worse progressively in the next decade. However, symptoms usually calm down after this progression period. Enlarged hands and feet in early adulthood or late childhood can be caused by different disorders, including excess $\mathrm{GH}$ production. In a young patient with acromegalic phenotype pathology of the somatotrophic axis must be excluded, before other differential diagnoses such as: pachydermoperiostosis or pseudoacromegaly(associated with severe insulin resistance) can be made. ${ }^{3}$ In patients with thickening of periosteum and skin, clubbing of fingers, acro-osteolysis, and alopecia, diagnosis of pachydermoperiostosis should be kept in mind. In such patients, acromegaly should be excluded, with normal levels of GH and IGF1.

The clinical features of pachydermoperiostosis vary. It is classified as the complete form (pachydermia, clubbing, and periostosis), the fruste form (prominent pachydermia with minimal skeletal changes) and the incomplete form which has no pachydermia. ${ }^{4}$ All our cases had thickened skin over hands, clubbing and radiological evidence of periostosis. Acro-osteolysis was seen in our patients which is the common x-ray finding of pachydermoperiostosis that affects the terminal phalanges of fingers. Joint effusions and arthritis have been reported in $20-40 \%$ of patients. ${ }^{5}$ Case 1 and 4 had typical arthritis and case 5 had severe joint pain. However, except case 4 who had drop foot no complications were noted in our cases including neuropathies due to compression of the spinal cord or nerve roots, entrapment neuropathies, and osteonecrosis of the femur.

As we know, there is limited knowledge for the current treatment modalities for pachydermoperiostosis. Usually, the first-line drugs are the conventional drugs like non-steroidal anti-inflammatory drugs and colchicines. ${ }^{6}$ Bisphosphonates were seen effective in reducing the pain and other symptoms related to hypertrophic osteoarthropathies. ${ }^{6-9}$ Pamidronate was reported to be one of promising drug which resulted in a significant pain relief in patients with hypertrophic osteoarthropathies. However, also zoledronic acid can be effective in hypertrophic osteoarthropathies as pain reliefer. ${ }^{10-13}$ The mechanism of action of bisphosphonates in hypertrophic osteoarthropathies 
remains unclear. But anti-resorptive and anti-inflammatory benefits are commonly accepted. ${ }^{10}$ In our patients with arthritis and severe joint pain, colchicine $3 \times 0,5 \mathrm{mg} /$ day, acemetacin $2 \times 60 \mathrm{mg} /$ day, and alendronic acid $1 \times 70 \mathrm{mg} /$ week treatment made remarkable recovery similar to our previous paper. ${ }^{14}$ Arthritis was recovered dramatically in a week, sedimentation rate and c- reactive protein were decreased (case 1: $12 \mathrm{~mm} / \mathrm{h}, 1.1 \mathrm{mg} / \mathrm{L}$ and case $4: 19 \mathrm{mg} / \mathrm{h}, 0.31 \mathrm{mg} / \mathrm{L}$, respectively). This clinical well-being continued in the control after 3 years for case 1, 1 year each for case 4 and 5 . Colchicine and alendronic acid treatment were stopped and only acemetacin treatment in case of need continued for case 1. Case 4 and 5 are still continuing the combination therapy. For other patients, without complaints except clubbing, we decided not to give any treatments.

\section{Conclusion}

In case of enlarging hands and feet, pachydermoperiostosis has to be kept in mind, especially as a differential diagnosis to acromegaly. Because the treatment of these disorders are totally different. Combination of acemetacin, colchicine, and alendronic acid seem to be effective in the treatment of pachydermoperiostosis, especially for acute symptoms which can be reduced quickly by the combination of these drugs.

\section{Acknowledgements}

None.

\section{Conflict of interest}

Authors declare there is no conflict of interest in publishing the article.

\section{References}

1. Favus MJ. Paget disease and other dysplasias of bone. In: Kasper DL, Braunwald E, Fauci AS, et al. editors. Harrison's Principles of Internal Medicine. 17th ed. New York: The McGraw-Hill Companies; 2008: 2414.

2. Gilliland BC. Relapsing polychondritis and other arthritides. In: Fauci AS, Braunwald E, Isselbacher KJ, et al. editors. Harrison's Principles of Internal Medicine. 14th ed. New York: McGraw-Hill Book Co; 1998:1951-63.

3. Flier JS, Moller DE, Moses AC, et al. Insulin-mediated pseudoacromegaly: Clinical and biochemical characterization of a syndrome of selective insulin resistance. J Clin Endocrinol Metab. 1993;76:1533-41.

4. Matucci-Cerinic M, Lotti T, Jajic I, et al. The clinical spectrum of pachydermoperiostosis (primary hypertrophic osteoarthropathy). Medicine (Baltimore). 1991;70(3):208-14.

5. Schumacher HR. Hypertrophic osteoarthropathy: Rheumatologic manifestations. Clin Exp Rheumatol. 1992;10:35-40.

6. Mauricio O, Francis L, Athar U, et al. Hypertrophic osteoarthropathy masquerading as lower extremity cellulitis and response to bisphosphonates. J Thorac Oncol. 2009;4:260-2.

7. Pineda C, Lavin MM. Hypertrophic osteoarthropathy. What a rheumatologist should know about this uncommon condition. Rheum Dis Clin N Am. 2013;39(2):383-400.

8. Jojima H, Kinoshita K, Naito M. A case of pachydermoperiostosis treated by oral administration of a bisphosphonate and arthroscopic synovectomy. Mod Rheumatol 2007;17:330-2.

9. Reid IR. Bisphosphonates: new indications and methods of administration. Curr Opin Rheumatol. 2003;15(4):458-63.

10. Jayakar BA, Abelson AG, Yao Q. Treatment of hypertrophic osteoarthropathy with zoledronic acid: case report and review of the literature. Semin Arthritis Rheum. 2011;41(2):291-6.

11. King MM, Nelson DA. Hypertrophic osteoarthropathy effectively treated with zoledronic acid. Clin Lung Cancer. 2008;9(3):179-82.

12. Thompson MA, Warner NB, Hwu WJ. Hypertrophic osteoarthropathy associated with metastatic melanoma. Melanoma Res. 2005;15(6):55961.

13. Zhang Q, Shen M, Yang B, et al. A complicated case of pachydermoperiostosis with spondyloarthritides: a case report. Journal of Medical Case Reports 2013;7:268-72.

14. İlhanlı İ. Pachydermoperiostosis: A Rare Clinical Entity. Turk J Phys Med Rehab. 2015;61:89-90. 